\title{
It All Just Clicks: Development of an Inpatient E-Consult Program
}

\author{
Nader Najafi, $\mathrm{MD}^{1 *}$, James D. Harrison, $\mathrm{PhD}^{1}$, Jonathan Duong, MD', \\ Anya Greenberg, MBA², Hugo Quinny Cheng, MD
}

\begin{abstract}
${ }^{1}$ Division of Hospital Medicine, University of California San Francisco, San Francisco, California; ${ }^{2}$ University of California San Francisco Medical
\end{abstract} Center, San Francisco, California.

\begin{abstract}
Although the use of electronic consultations (e-consults) in the outpatient setting is commonplace, there is little evidence of their use in the inpatient setting. Often, the only choice hospitalists have is between requesting a time-consuming in-person consultation or requesting an informal, undocumented "curbside" consultation. For a new, remote hospital in our healthcare system, we developed an e-consult protocol that can be used to address simple consultation questions. In the first year of the program, 143 e-consults occurred; the top 5 consultants were infectious disease, he-
\end{abstract}

matology, endocrinology, nephrology, and cardiology. Over the first 4 months, no safety issues were identified in chart review audits; to date, no safety issues have been identified through the hospital's incident reporting system. In surveys, hospitalists were universally pleased with the quality of e-consult recommendations, though only $43 \%$ of consultants agreed. With appropriate care for patient selection, e-consults can be used to safely and efficiently provide subspecialty expertise to a remote inpatient site. Journal of Hospital Medicine 2017;12:332-334. (C 2017 Society of Hospital Medicine
Electronic consultation (e-consult) in the outpatient setting allows subspecialists to provide assessment and recommendations for patients without in-person visits. ${ }^{1}$ An e-consult is an asynchronous communication that uses the electronic medical record (EMR) and typically involves an electronic order from a requesting provider and an electronic note from a consulting provider. The initial motivation for developing this consultation modality was to improve access to subspecialty care for patients in the primary care setting, and findings of studies at several sites support this claim. ${ }^{1-4}$ In addition, e-consult may also reduce cost because converting unnecessary face-to-face encounters into e-consults reduces patients' travel costs and healthcare organizations' expensive subspecialty clinic time..$^{3,5}$ Moreover, instead of addressing less complex clinical questions in informal, undocumented face-to-face or telephone "curbside" consultations with specialists, providers can instead ask for e-consults and thereby ensure thorough chart review and proper documentation. ${ }^{6}$

Use of e-consults in the inpatient setting is relatively novel. ${ }^{7}$ In addition to having the advantages already mentioned, e-consults are faster than in-person bedside consultations and may be beneficial in the fast-moving inpatient care setting. Finally, healthcare systems with multiple hospital sites may not have the capacity to physically locate subspecialists at each site, which makes e-consults attractive for avoiding unnecessary travel time.

In this article, we describe how we developed an inpatient

\footnotetext{
*Address for correspondence and reprint requests: Nader Najafi, MD, Division of Hospital Medicine, University of California San Francisco, 505 Parnassus Ave, M1283, San Francisco, CA 94143-0131; Telephone: 415-476-2223; Fax: 415-502-1963; E-mail: nader.najafi@ucsf.edu
}

Received: August 11, 2016; Revised: October 22, 2016; Accepted: November 9, 2016

2017 Society of Hospital Medicine DOI 10.12788/jhm.2740 e-consult protocol for a new, remote hospital within our healthcare system and explore data on safety and physician attitudes after e-consult implementation.

\section{METHODS}

The Institutional Review Board of the University of California San Francisco (UCSF) approved this study.

\section{Setting}

In February 2015, UCSF opened a new hospital in the Mission Bay neighborhood of San Francisco, 4 miles from the existing hospital. The new hospital is home to several adult inpatient services: urology, otolaryngology, colorectal surgery, obstetrics, and gynecologic surgery. A hospitalist is on-site 24 hours a day to provide consultation for these services around issues that relate to internal medicine. A hospitalist who requires subspecialty expertise to answer a clinical question can request a consultation by in-person visit, video telemedicine, or e-consult, each of which is available 24/7. Almost all of the medicine subspecialists work on the existing campus, not in Mission Bay.

\section{Protocol Development and Implementation}

The protocol for the e-consult program was developed over several months by an interdisciplinary group that included 3 hospitalists, 1 obstetrician, 1 project manager, and 1 informaticist. The group outlined the process for requesting and completing an e-consult (Figure), designed a note template for consultants to use for EMR documentation, conducted outreach with subspecialty groups to discuss the protocol, and developed an EMR report to track e-consult use and content over time. As our medical center does not bill payers for inpatient e-consults, e-consult note tracking is used to provide reimbursement internally, from the medical center to the respective departments of the consultants. Reim- 


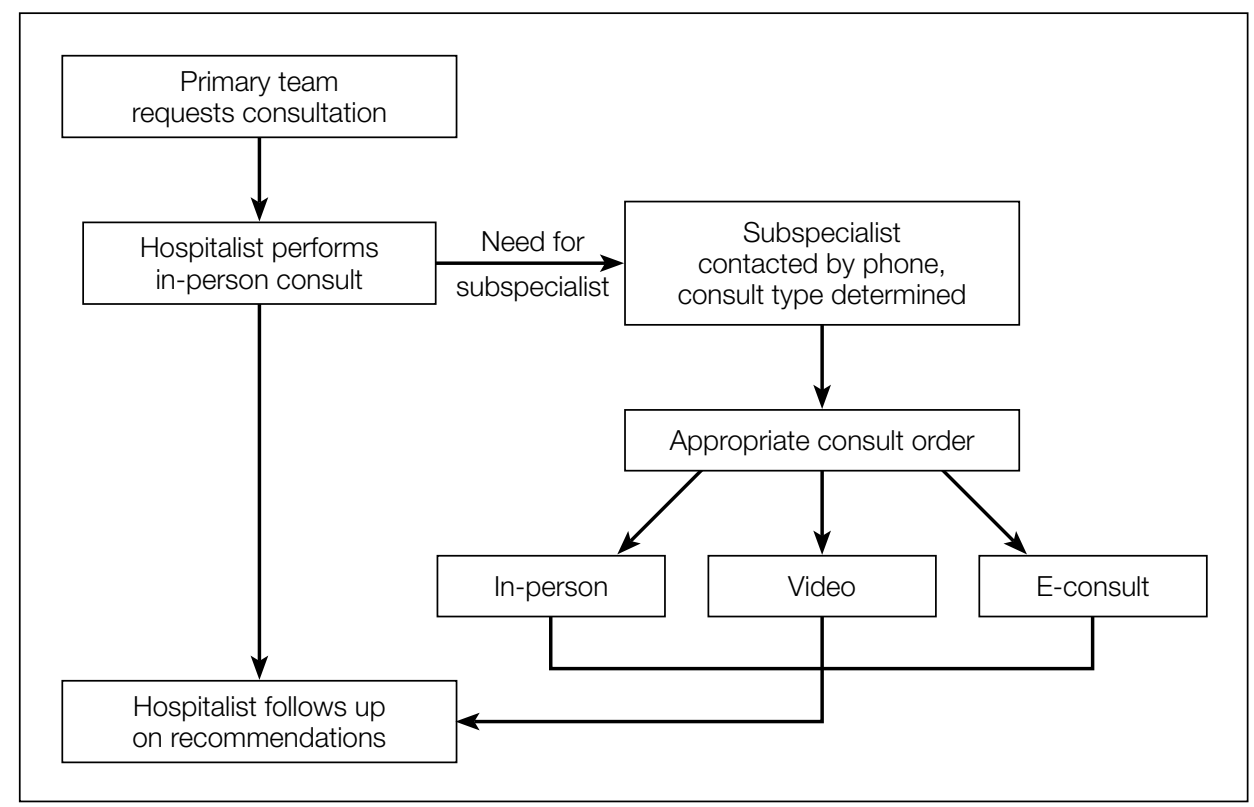

FIG. Process for requesting and completing an e-consult. Order not mandatory.
Eight months after the program started, we assessed experience by electronically surveying the 9 hospitalists and 11 consultants who had requested or performed at least 2 e-consults. ${ }^{8}$ Survey items were measured on a 5 -point Likert scale: strongly disagree to strongly agree. The items, which related to ease of calling for a consultation, quality of e-consults, impact on clinical care, safety concerns, and satisfaction, were inspired by themes identified in a systematic review of the literature on e-consults in the outpatient setting. ${ }^{2}$ We sent 2 reminders to responders. Data were summarized using descriptive statistics. Analysis was performed in SPSS version 22.0 (IBM).

\section{RESULTS}

bursement is made at a set rate per e-consult note, with the rate set to approximate the reimbursement of a low-acuity in-person consult on the main campus.

The workflow of an e-consult is as follows: (1) When a primary team requires a consultation on an issue that falls within the purview of internal medicine, it pages the onsite hospitalist. (2) The hospitalist accepts the consultation by phone, reviews the chart, and examines the patient. (3) If the hospitalist requires subspecialty assistance to answer a clinical question, he or she contacts the appropriate subspecialty service by pager. (4) The subspecialist speaks with the hospitalist about the consultation question, and together they decide if an e-consult is appropriate, based on the complexity of the clinical scenario. (5) The subspecialist reviews the patient's chart and documents their care plan recommendations in an e-consult note. Consultants can use e-consult for both initial and follow-up assessment, and there is no strict requirement that they also contact the hospitalist or the primary team by phone in addition to consultation note. Given their novelty, almost all e-consults are performed by subspecialist attendings, not residents or fellows.

\section{Evaluation}

Each month, we tracked e-consult use using an EMR report built as part of the implementation of the program. For the first four months of implementation, every patient who received an e-consult also had a manual chart review of the period around the e-consult, performed by a hospitalist, in order to audit for any potential safety issues. These issues included, for example, an e-consult performed for a patient whose complexity or severity of illness was felt to be too great to defer an in-person visit, or a patient who received e-consult recommendations that were significantly retracted in a follow-up in-person note.
There were 143 initial subspecialty consultations by e-consult between program launch in February 2015 and manuscript preparation in February 2016, an average of 11 e-consults per month. There were 313 total e-consult notes (these included both initial and follow-up e-consult notes). By comparison, 240 initial in-person consultations occurred during the same period, and there were 435 total in-person consultation notes (46\% new or initial notes, $54 \%$ follow-up notes). The top 5 subspecialties by volume of e-consults were infectious disease (35\%), hematology (20\%), endocrinology (14\%), nephrology (13\%), and cardiology (8\%). For reference, e-consults are also available from psychiatry, neurology, oncology, gastroenterology, pulmonology, and rheumatology. Percentage of consultations performed during daytime hours (defined as 8 a.m. to 5 p.m.) was $92 \%$ for e-consults and $96 \%$ for in-person consultations.

There were no e-consult-related patient safety issues reported through the medical center's incident reporting system during the study period. There were also no patient safety issues identified in the manual audits of 80 charts during the first 4 months of the program.

Seven (78\%) of 9 hospitalists and 7 (64\%) of 11 consultants completed the survey. Both groups agreed that e-consults were easy to use and efficient (Table). All hospitalists were satisfied with the quality of e-consult recommendations, but only 3 (43\%) of the 7 consultants agreed they could provide high-quality consultation by e-consult. In their comments, 2 consultants expressed concerns. One concern was about missing crucial information by performing only a chart review, and the other was about being tempted to perform an e-consult simply because it is expedient.

\section{DISCUSSION}

Although use of e-consults in the outpatient setting is relatively commonplace, our program represents a novel use 
TABLE. Results of E-Consult Survey Given to Hospitalists and Consultants

\begin{tabular}{|c|c|c|c|}
\hline Hospitalists $(n=7)$ & $\begin{array}{c}\text { Agree or } \\
\text { Strongly Agree, } \\
\mathrm{n}(\%)\end{array}$ & Consultants $(n=7)$ & $\begin{array}{c}\text { Agree or } \\
\text { Strongly Agree, } \\
\mathrm{n}(\%)\end{array}$ \\
\hline It is easy to request an e-consult & $5(71)$ & It is easy to provide an e-consult & $4(57)$ \\
\hline I am satisfied with the quality of e-consult recommendations & $7(100)$ & $\begin{array}{l}\text { The referring provider adequately communicates the e-consult question } \\
\text { and important clinical information }\end{array}$ & $5(71)$ \\
\hline $\begin{array}{l}\text { It is less safe to accept recommendations from an e-consult than in an } \\
\text { in-person consult }\end{array}$ & $1(14)$ & $\begin{array}{l}\text { It is less safe to provide recommendations through an e-consult than } \\
\text { in an in-person consult }\end{array}$ & $2(29)$ \\
\hline I am satisfied with the turnaround time for e-consults & $6(86)$ & I can provide a high-quality consultation through e-consult & $3(43)$ \\
\hline An e-consult usually eliminates the need for an in-person consult & $5(71)$ & $\begin{array}{l}\text { It is more efficient for me to perform an e-consult than an } \\
\text { in-person consult }\end{array}$ & $6(86)$ \\
\hline Recommendations from an e-consult often modify my care plan & $6(86)$ & The e-consult program should be expanded to other sites at my institution & $3(43)$ \\
\hline Overall I am satisfied with the e-consult program & $6(86)$ & & \\
\hline
\end{tabular}

of e-consults in safely and efficiently providing subspecialty consultation to inpatients at a remote hospital.

For hospitalists, an e-consult system offers numerous benefits. Clinical questions beyond an internists' scope of practice come up often, and simple questions might traditionally result in an informal curbside consult. While a curbside consult provides answers faster than an in-person visit, it creates risks for the requesting hospitalists: the consultants only know what they are told, whether the information is incomplete or erroneous; their opinions are given without documentation or compensation, which reduces a sense of accountability; and the lack of documentation does not allow their advice to persist in the chart as a reference for future providers. ${ }^{9}$ Our e-consult program solves these problems by requiring that consultants perform chart review and provide documentation as well as obligating the medical center to pay a small compensation to consultants for their time. We hope this lowers the bar to requesting consultation for remote sites, where the alternative would be burdensome travel time to do an in-person visit.

In our study, hospitalists were universally pleased with the quality of e-consult recommendations, but only $43 \%$ of consultants agreed. These findings correlate with the literature on e-consults in the outpatient setting. ${ }^{2}$ Unfortunately, our survey comments did not shed further light on this sentiment. In the outpatient literature, consultants were most concerned with having a clear clinical question, facing the liability of providing recommendations without performing an examination, and receiving appropriate compensation for answering e-consults.

The generalizability of our program findings is limited most significantly by the particular arrangement of our clinical services: Our remote site is home to a select group of adult inpatient services, a hospitalist is available on-site for these services 24 hours a day, and the distance to the remote site can be overcome with modest effort should a patient require an in-person visit in the initial or follow-up period. The generalizability of our safety findings is limited by the use of a single reviewer for chart auditing.

Given the rise of accountable care organizations and the prevalence of hospital mergers in the healthcare landscape, we believe that healthcare systems that operate remote sites under constrained budgets could look to e-consults to more cost-effectively extend subspecialty expertise across the inpatient enterprise. With improvements in health information exchange, it may also become feasible for consultants to offer e-consults to hospitals outside a medical center's network. Our study showed that inpatient e-consult programs can be developed and implemented, that they appear not to pose any significant safety issues, and that they can facilitate delivery of timely clinical care.

\section{Acknowledgment}

The authors thank Raphaela Levy-Moore for creating and implementing the e-consult note template for our electronic medical record.

Disclosure: Nothing to report.

\section{References}

1. Chen AH, Murphy EJ, Yee HF Jr. eReferral-a new model for integrated care N Engl J Med. 2013;368(26):2450-2453.

2. Vimalananda VG, Gupte G, Seraj SM, et al. Electronic consultations (e-consults) to improve access to specialty care: a systematic review and narrative synthesis. J Telemed Telecare. 2015;21(6):323-330.

3. Kirsh S, Carey E, Aron DC, et al. Impact of a national specialty e-consultation implementation project on access. Am J Manag Care. 2015;21(12):e648-e654.

4. Bergman J, Neuhausen K, Chamie K, et al. Building a medical neighborhood in the safety net: an innovative technology improves hematuria workups. Urology. 2013;82(6):1277-1282.

5. Wasfy JH, Rao SK, Chittle MD, Gallen KM, Isselbacher EM, Ferris TG. Initial results of a cardiac e-consult pilot program. J Am Coll Cardiol. 2014;64(24): 2706-2707.

6. Perley CM. Physician use of the curbside consultation to address information needs: report on a collective case study. J Med Libr Assoc. 2006;94(2):137-144.

7. Gupte G, Vimalananda V, Simon SR, DeVito K, Clark J, Orlander JD. Disruptive innovation: implementation of electronic consultations in a Veterans Affairs health care system. JMIR Med Inform. 2016;4(1):e6.

8. REDCap. Vanderbilt University website. http://www.project-redcap.org. 2015. Accessed March 3, 2016.

9. Burden M, Sarcone E, Keniston A, et al. Prospective comparison of curbside versus formal consultations. J Hosp Med. 2013;8(1):31-35. 